\title{
Bile Salt Homeostasis in Normal and Bsep Gene Knockout Rats with Single and Repeated Doses of Troglitazone ${ }^{[\mathrm{S}}$
}

\author{
Yaofeng Cheng, Shenjue Chen, Chris Freeden, Weiqi Chen, Yueping Zhang, \\ Pamela Abraham, David M. Nelson, W. Griffith Humphreys, Jinping Gan, and Yurong Lai \\ Pharmaceutical Candidate Optimization, Bristol-Myers Squibb, Princeton, New Jersey
}

Received April 23, 2017; accepted June 22, 2017

\begin{abstract}
The interference of bile acid secretion through bile salt export pump (BSEP) inhibition is one of the mechanisms for troglitazone (TGZ)induced hepatotoxicity. Here, we investigated the impact of single or repeated oral doses of TGZ (200 mg/kg/day, 7 days) on bile acid homoeostasis in wild-type (WT) and Bsep knockout (KO) rats. Following oral doses, plasma exposures of TGZ were not different between WT and KO rats, and were similar on day 1 and day 7 . However, plasma exposures of the major metabolite, troglitazone sulfate (TS), in KO rats were 7.6- and 9.3-fold lower than in WT on day 1 and day 7 , respectively, due to increased TS biliary excretion. With Bsep KO, the mRNA levels of multidrug resistance-associated protein 2 (Mrp2), Mrp3, Mrp4, Mdr1, breast cancer resistance protein (Bcrp), sodium taurocholate cotransporting polypeptide,
\end{abstract}

small heterodimer partner, and Sult2A1 were significantly altered in KO rats. Following seven daily TGZ treatments, Cyp7A1 was significantly increased in both WT and KO rats. In the vehicle groups, plasma exposures of individual bile acids demonstrated variable changes in KO rats as compared with WT. WT rats dosed with TGZ showed an increase of many bile acid species in plasma on day 1 , suggesting the inhibition of Bsep. Conversely, these changes returned to base levels on day 7 . In $\mathrm{KO}$ rats, alterations of most bile acids were observed after seven doses of TGZ. Collectively, bile acid homeostasis in rats was regulated through bile acid synthesis and transport in response to Bsep deficiency and TGZ inhibition. Additionally, our study is the first to demonstrate that repeated TGZ doses can upregulate Cyp7A1 in rats.

\section{Introduction}

Drug-induced liver injury (DILI) is one of most frequently observed adverse effects in clinical practice and can lead to acute liver failure and liver transplant (Leise et al., 2014). Meanwhile, many drugs have been withdrawn from the market due to severe DILI, such as iproniazid, ticrynafen, benoxaprofen, bromfenac, troglitazone (TGZ), and ximelagatran (Lammert et al., 2008). Causes of DILI are complicated, and a single factor or multiple combinations could contribute to these causes, including drug physicochemical properties, pharmacokinetics, reactive metabolites, oxidative stresses, mitochondrial liabilities, immune responses, and drug endogenous molecule interactions (Hussaini and Farrington, 2007).

Transporters are a class of membrane proteins which are critical for normal cell functions by regulating the intake of nutrients and physiologic chemicals and the elimination of waste and toxins. Alteration of transporter activities can cause severe organ injuries or systemic toxicities in humans (Cheng et al., 2016a). For example, bile salt export pump (BSEP) is an

https://doi.org/10.1124/jpet.117.242370.

S This article has supplemental material available at jpet.aspetjournals.org. adenosine triphosphate (ATP)-dependent transporter predominantly expressed on the canalicular membrane of hepatocytes, where its major function is to mediate the efflux of bile acids from hepatocytes to the bile duct to maintain normal bile acid homeostasis in the body (Meier et al., 1984; Stieger et al., 1992; Gerloff et al., 1998). Impaired BSEP function can lead to intrahepatic cholestasis in humans as a result of intracellular accumulation of bile acids in hepatocytes. Several BSEP gene mutations are confirmed to be associated with progressive familial intrahepatic cholestasis type 2 in humans, a progressive cholestasis usually necessitating liver transplantation (Jansen et al., 1999; Lang et al., 2007; Ho et al., 2010). On the other hand, inhibition of BSEP activity by drugs can result in similar consequences. For example, TGZ-induced hepatotoxicity observed in the clinic is related to, at least partially, the inhibition of BSEP by TGZ and its sulfate-conjugated metabolite (Izumi et al., 1996; Funk et al., 2001a,c; Smith, 2003). Systemic investigations of hundreds of drugs have demonstrated the correlation between BSEP inhibition and DILI (Morgan et al., 2010, 2013; Pedersen et al., 2013).

As drugs and/or metabolites with the capability to inhibit BSEP have the potential to cause DILI, a variety of in vitro

ABBREVIATIONS: ALT, alanine transaminase; $\mathrm{AUC}_{0-24}$, area under the curve from time zero to 24 hours post dosing; Baat, bile acid-CoA:amino acid $\mathrm{N}$-acyltransferase; Bcrp, breast cancer resistance protein; Bsep, bile salt export pump; CA, cholic acid; CDCA, chenodeoxycholic acid; DCA, deoxycholic acid; DILI, drug-induced liver injury; Fxr, farnesoid X receptor; GCA, glycocholic acid; GCDCA, glycochenodeoxycholic acid; GDCA, glycodeoxycholic acid; HPLC, high-performance liquid chromatography; KO, knockout; $\alpha \mathrm{MCA} \alpha$-muricholic acid; $\beta \mathrm{MCA}, \beta$-muricholic acid; Mrp, multidrug resistance-associated protein; Ntcp, sodium taurocholate cotransporting polypeptide; PPAR, peroxisome proliferator-activated receptor; Shp, TCA, taurocholic acid; TCDCA, taurochenodeoxycholic acid; TDCA, taurodeoxycholic acid; TGZ, troglitazone; T $\alpha$ MCA, tauro- $\alpha$-muricholic acid; T $\beta$ MCA, tauro- $\beta$-muricholic acid; TS, troglitazone sulfate; WT, wild type; ZFN, zinc finger nuclease. 
assays have been developed in early drug discovery to screen BSEP inhibition, an effort to reduce the risk of hepatotoxicity in patients (Cheng et al., 2016b). However, translating in vitro BSEP inhibition data directly to human DILI outcomes remains challenging. Mice and rats have gene sequences of Bsep that are conservative to human orthologs, but they are more tolerable than humans with respect to BSEP impairment-related hepatotoxicity (Noe et al., 2001). For example, while humans with BSEP polymorphisms can develop progressive familial intrahepatic cholestasis type 2, Bsep knockout (KO) mice only display mild nonprogressive intrahepatic cholestasis (Wang et al., 2001). Drugs such as bosentan and TGZ are known to cause DILI in humans, but are well tolerated in rodents at much higher plasma exposures. One possible reason is that the bile acid pool in rodents is more hydrophilic and less toxic (Perwaiz et al., 2003). Additionally, it has been reported that compensatory pathways in rodents are available to maintain bile acid homeostasis when there is a lack of Bsep activity (Wang et al., 2009). As such, characterizing the mechanisms associated with bile acid homeostasis can help us understand the rodent capability to handle drugs that can cause DILI in humans.

Previously, we reported a Bsep KO rat model in which Bsep was knocked out by zinc finger nuclease technology in Sprague-Dawley rats (Cheng et al., 2016b). This Bsep KO rat model showed a normal appearance and regular activity. The bile flow was within the normal range of reference values. However, bile acid profiles were altered in this Bsep KO rat model, and the liver has a reduced capacity to excrete administered taurocholic acid (TCA). In the present study, we investigated the effect of single or repeated oral doses of TGZ on bile salt homeostasis in rats. Following the administration of TGZ at a dose of $200 \mathrm{mg} / \mathrm{kg} /$ day for up to 7 days, individual bile acid profiles were characterized in wild-type (WT) and Bsep KO rats. Additionally, gene expressions of liver transporters, enzymes, and nuclear factors that are associated with bile acid disposition were also evaluated to compare with the bile acid profiles.

\section{Materials and Methods}

Chemicals and Materials. TGZ was synthesized by BristolMyers Squibb (New Brunswick, NJ). Troglitazone sulfate (TS) and pioglitazone were purchased from Sigma-Aldrich (St. Louis, MO). Chenodeoxycholic acid (CDCA), cholic acid (CA), deoxycholic acid (DCA), glycocholic acid (GCA), glycodeoxycholic acid (GDCA), glycochenodeoxycholic acid (GCDCA), TCA, taurodeoxycholic acid (TDCA), and taurochenodeoxycholic acid (TCDCA) were purchased from Sigma-Aldrich. $\alpha$-Muricholic acid ( $\alpha$ MCA), tauro- $\alpha$-muricholic acid (T $\alpha$ MCA), $\beta$-muricholic acid ( $\beta$ MCA), and tauro- $\beta$-muricholic acid (T $\beta$ MCA) were purchased from Steraloid Inc. (Newport, RI). Deuterium-labeled deoxycholic acid was purchased from CDN Isotopes (Quebec, Canada). Adenosine 3'-phosphate-5'-phosphosulfate and alamethicin were obtained from Sigma-Aldrich.

Study Design. WT male Sprague-Dawley rats and male Bsep KO Sprague-Dawley rats were obtained from SAGE Laboratories (St. Louis, MO). All animals were maintained in the animal facility (Hopewell, $\mathrm{NJ})$ with controlled temperature $\left(25^{\circ} \mathrm{C}\right)$, humidity (40-60\%), and light/dark cycle (12 hours). The animal care and study protocols were approved by the Animal Care and Use Committee at Bristol-Myers Squibb (Hopewell, NJ). Rats were randomly assigned to four groups ( $n=5-6$ each group): TGZ ( $200 \mathrm{mg} / \mathrm{kg}, 2 \mathrm{ml} / \mathrm{kg})$ in KO rats, TGZ (200 mg/kg, $2 \mathrm{ml} / \mathrm{kg})$ in WT rats, vehicle $(2 \mathrm{ml} / \mathrm{kg})$ in KO rats, and vehicle $(2 \mathrm{ml} / \mathrm{kg})$ in WT rats. TGZ was prepared as suspension in the dosing vehicle of $0.15 \%$ docusate sodium/Polyvinylpyrrolidone-K30/ $\mathrm{H}_{2} \mathrm{O}(0.15 / 2 / 97.85 \%)$ at a concentration of $100 \mathrm{mg} / \mathrm{ml}$. The particle size of $90 \%$ TGZ was less than $8 \mu \mathrm{m}$. The purity of TGZ was greater than $99 \%$, and the formulation was stable for a week as checked by highperformance liquid chromatography (HPLC).

After at least a week of acclimation, the rat jugular vein was cannulated for blood collection. On the following day, rats received a once-daily oral dose through oral gavage for 7 days. Plasma samples were collected at $0,0.5,1,2,4,7$, and 24 hours after the first and seventh doses. Serum was collected at 0 hours (predose) on day 1 and 24 hours on day 7 . Withdrawn blood $(\sim 300 \mu \mathrm{l})$ was replaced with an equal volume of saline solution. At 24 hours after the seventh dose, animals were euthanized by $\mathrm{CO}_{2}$ inhalation and liver was collected from each animal. Plasma and liver samples were stored at $-80^{\circ} \mathrm{C}$ until analysis.

TGZ and TS Quantitation. All samples were removed from $-80^{\circ} \mathrm{C}$ freezers and thawed, and then processed and analyzed immediately to minimize the degradation of the test articles. Plasma samples $(30 \mu \mathrm{l})$ were extracted with $60 \mu \mathrm{l}$ of acetonitrile containing pioglitazone as the internal standard. Liver samples were homogenized with $10 \times$ volume of blank rat plasma and then processed the same as plasma samples. Reference standards of TGZ and TS were spiked in control rat plasma and then processed the same as testing samples. The mixture was then vortexed and filtered through filter plates. With the addition of $60 \mu \mathrm{l}$ of $\mathrm{H}_{2} \mathrm{O}$, an aliquot of the samples $(5 \mu \mathrm{l})$ was injected into a Shimadzu LC-10AD HPLC system (Shimadzu Scientific Instruments, Columbia, MD) connected with an Atlantis C18 column $(5 \mu \mathrm{m}, 2.1 \times 50 \mathrm{~mm}$, Waters, Milford, MA). The initial mobile phase of $5 \%$ solvent B was changed to $20 \%$ solvent B over 1.5 minutes, then to $95 \%$ solvent $\mathrm{B}$ over 1 minute with linear gradients; solvent $\mathrm{A}$ is $0.1 \%$ formic acid in water, and solvent $B$ is acetonitrile. The flow rate was $0.3 \mathrm{ml} / \mathrm{min}$. The HPLC eluate was introduced into a Sciex Q-Trap 4000 mass spectrometer (AB Sciex, Framingham, MA) equipped with an electrospray ionization source operating in negative-ion mode. The specific transitions for monitored analytes were TGZ $(\mathrm{m} / \mathrm{z} 440.1 \rightarrow 397.2)$, $\mathrm{TS}(\mathrm{m} / \mathrm{z} 520.1 \rightarrow 440.4)$, and pioglitazone $(\mathrm{m} / \mathrm{z} 355 \rightarrow 311)$. In the evaluation of this analytical method, no carryover was observed between two sample injections. The lower limits of quantitation were found to be 30 and $10 \mathrm{nM}$ for TGZ and TS, respectively. The ion responses were reliable up to $10 \mu \mathrm{M}$ within $20 \%$ variance for both TGZ and TS. Concentrations of TGZ and TS were determined based on their respective standard curves, and samples with a concentration greater than the highest standard concentration were diluted and reanalyzed.

TS Formation in Liver S9 Incubation. To compare the enzymatic activities toward formation of TS, the relative amount of TS was quantified following incubation with TGZ and liver S9 fraction from WT and KO rats treated with vehicle. Liver S9 was prepared by centrifuging the liver tissue (homogenized with $5 \times$ volume of phosphate buffer, $\mathrm{pH} 7.4$ ) at $10,000 \mathrm{~g}$ for 30 minutes at $4^{\circ} \mathrm{C}$. The protein concentration was measured using a BCA protein assay (Thermo Scientific, Rockford, IL). The in vitro reaction was carried out in Tris-Cl buffer ( $\mathrm{pH} 7.4,100 \mathrm{mM})$ containing adenosine 3 '-phosphate-5' phosphosulfate $(\sim 0.1 \mathrm{mM})$, alamethicin $(25 \mathrm{mg} / \mathrm{ml})$, and TGZ $(10 \mu \mathrm{M})$. The mixture was preincubated for 5 minutes at $37^{\circ} \mathrm{C}$ and then the reaction was initiated by adding $\mathrm{S} 9$ fraction $(1 \mathrm{mg} / \mathrm{ml})$. An aliquot of samples $(100 \mu \mathrm{l})$ was collected at $5,15,30$, and 60 minutes and extracted with $200 \mu \mathrm{l}$ of acetonitrile containing tolbutamide (100 nM), followed with filtration through filter plates. The supernatant was brought to near dryness (not totally dry) under $\mathrm{N}_{2}$ and then reconstituted in $200 \mu \mathrm{l}$ of $\mathrm{H}_{2} \mathrm{O}$ with $0.1 \%$ formic acid. Samples were analyzed using liquid chromatography-tandem mass spectrometry as described above. The specific multiple reaction monitoring transition for tolbutamide was $269.1 \rightarrow 169.8$. The relative amount of TS was quantified based on the peak area ratio between TS and tolbutamide.

Liver RNA Isolation and Real-Time Polymerase Chain Reaction Analysis. The gene expression of selected liver transporters, metabolic enzymes, and nuclear receptors (as listed in Table 1) was measured in liver samples collected from Bsep WT and 
TABLE 1

List of primers and probes for real-time PCR

\begin{tabular}{|c|c|c|c|}
\hline Gene & Primer or Probe Sequence & Position & Accession Number \\
\hline Peptidylprolyl isomerase & GGTCGCGTCTGCTTCGA & F94 & NM_017101 \\
\hline (PPIA) & CTGGAATAATTCTGTGAAAGGAGGAA & $\mathrm{R} 217$ & \\
\hline Multidrug resistance-associated protein 2 & CGAGACGGATAGCCTCATTCA & F4489 & NM_012833 \\
\hline (Mrp2) & ATCTTCCCGTTGTCTAGGACCAT & $\mathrm{R} 4617$ & \\
\hline Multidrug resistance-associated protein 3 & CCCACCGGCTCAACACA & F4478 & NM 080581 \\
\hline (Mrp3) & ATGCCTCCAGCTGCAATGA & $\mathrm{R} 4583$ & \\
\hline Multidrug resistance-associated protein 4 & ATTGTGGGAAGAACTGGAGCTGGA & F3217 & NM_133411 \\
\hline (Mrp4) & TGGTTCCAGTGAACAGGACAGGTT & R3368 & \\
\hline P-glycoprotein & TACACAGACCGTCAGCGACA & F323 & NM_012623 \\
\hline (P-gp) & GCCAGGCACCAAAGTGAAAC & R445 & \\
\hline Sodium/bile acid cotransporter & TCAAGCCTCCAAAGGACCAA & F1050 & NM 017047 \\
\hline (Ntcp) & GCCATTAGGGGAAGGACCAG & R1183 & \\
\hline Organic anion-transporting polypeptide $1 \mathrm{~A} 1$ & AATACCTGGAACAGCAGTATGGAAA & F1108 & NM_017111 \\
\hline (Oatp1A1) & CCGAGGCATATGGGAGGTAA & R1192 & \\
\hline Farnesoid X receptor & TGGGAATGTTGGCTGAATGTTT & F731 & NM_021745.1 \\
\hline (Fxr) & TGCATAGCTTGGTCGTGGAG & R880 & \\
\hline Small heterodimer partner & CACTATCCTCTTCAACCCAGATGT & F556 & NM 057133.1 \\
\hline (Shp) & GGCTCCAGGACTTCACACAAT & R657 & \\
\hline Bile acid-CoA:amino acid $N$-acyltransferase & GTCGAACTACGGTTTTGGCG & F137 & NM_017300.2 \\
\hline (Baat) & CCGGATATGCACAGGCTCAT & R257 & \\
\hline Cytochrome P450 7A1 & TTGGATCAAGTGCAACTGAATGA & F1038 & NM_012942 \\
\hline (Cyp7A1) & GGAACCGTCCTCAAGATGGA & R1169 & \\
\hline Bile salt sulfotransferase & TGGTTCCTCAAAGGAAATGTTCTA & F488 & NM_131903.1 \\
\hline (Sult2A1) & AGAAGTTGTCCCATTCTCGCA & R572 & \\
\hline Bile salt export pump & ACGGAACAGTCTGTGGGTTG & F636 & NM 031760 \\
\hline (Bsep) & GACTCCTGCGTAGATGCCAG & R706 & \\
\hline Bsep WT and KO & TGCTTTCGAATCAGATGGATCACAT & F316 & \\
\hline Bsep WT and KO & TCAAAGAAGCCAACTCGAATGTCT & R408 & \\
\hline Bsep knockout probe & CCTCCTTCATGTAACCTTG & P375 & \\
\hline Bsep wild-type probe & CCCTCCTTCATCTTATCTTGTAA & P384 & \\
\hline
\end{tabular}

KO rats dosed with vehicle or TGZ for 7 days. For mRNA isolation, frozen liver was first homogenized in TRIzol (Life Technologies, Grand Island, NY) using 5-mm-diameter stainless steel beads with TissueLyser (Qiagen, Valencia, CA). Total RNA was purified using the RNeasy Mini Kit (Qiagen) including the on-column DNase treatment following the manufacturer's protocol. The quality and quantity of liver total RNA were evaluated with a NanoDrop 8000 (Thermo Scientific, Wilmington, DE). cDNA was synthesized from $2 \mu \mathrm{g}$ of total RNA using SuperScript III Reverse Transcriptase (Life Technologies).

Real-time polymerase chain reaction (PCR) was performed in duplicate on the 384-well block format ViiA 7 Real-Time PCR System using Power SYBR Green PCR Master Mix (Life Technologies). PCR was performed with the default thermal profile using diluted cDNA equivalent to $16 \mathrm{ng}$ of total RNA as the PCR template. The assayed genes with full names, GeneBank accession numbers, and PCR primer sequences are provided in Table 1 . The mRNA expression of each gene was normalized to the housekeeping gene peptidylprolyl isomerase A.

To confirm the Bsep knockout, two TaqMan probes at the zinc finger nuclease (ZFN)-targeted mutation site were designed, one for the intact cDNA sequence and the other for the eight-nucleotide deleted sequence. PCR was performed as described earlier, except using TaqMan Gene Expression Master Mix (Life Technologies).

Full-Length Bsep cDNA Sequence Analysis. To further verify that the ZFN-targeted eight-nucleotide mutation region is intact in WT rats and is deleted in Bsep KO rats, full-length rat Bsep cDNA from all rats was sequenced. PCR amplification was carried out using Platinum Taq High Fidelity DNA polymerase (Life Technologies) with the following thermocycling profile: $94^{\circ} \mathrm{C}$ for 2 minutes, followed by 35 cycles of $94^{\circ} \mathrm{C}$ for 15 seconds, $59^{\circ} \mathrm{C}$ for 30 seconds, and $68^{\circ} \mathrm{C}$ for 1 minute, and a final $68^{\circ} \mathrm{C}$ for 7 minutes. PCR amplicon products were analyzed by gel electrophoresis, and the expected size bands were identified. The PCR products from each rat liver were sequenced to confirm sequence identity.

Serum Chemistry. Serum chemistry tests were conducted for samples collected predose and 24 hours after the last dose. The samples were analyzed using the Siemens Advia 1800 automated chemistry instrument (Siemens Medical Solutions, Malvern, PA), and the tests included aspartate transaminase, alanine transaminase (ALT), alkaline phosphatase, total bilirubin, blood urea nitrogen, creatinine, cholesterol, triglycerides, and albumin.

Bile Acid Quantitation. The concentrations of bile acids were determined in all plasma samples collected on day 1 and day 7 , and in liver samples at the end of experiments. The monitored bile acids included CA, DCA, CDCA, GCA, GDCA, GCDCA, TCA, TDCA, TCDCA, $\alpha$ MCA, $\beta$ MCA, T $\alpha$ MCA, and T $\beta$ MCA. The analytical methods were the same as reported previously (Cheng et al., 2016b).

Data Analysis. The pharmacokinetic parameters of TGZ and TS in rat plasma were derived from plasma concentration versus time data with a noncompartment model using Phoenix WinNonlin 6.3 (Certara, Princeton, NJ). The plasma exposure of individual bile acids was determined as the area under the plasma concentration-time curve from time zero to 24 hours $\left(\mathrm{AUC}_{0-24}\right)$ using noncompartmental methods. All data here are reported as the mean \pm S.D., or as otherwise noted. The statistical test was conducted using one-way analysis of variance with Fisher's Least Significant Difference postanalysis using GraphPad Prism 7 (GraphPad Software, La Jolla, CA). Differences were considered significant when $p<0.05$.

\section{Results}

Following the first oral dose, TGZ was rapidly absorbed, and the time of maximum plasma concentration $\left(\mathrm{T}_{\max }\right)$ was 1.8 and 1.5 hours in WT and KO rats, respectively (Fig. 1A). The peak plasma concentration $\left(\mathrm{C}_{\max }\right), \mathrm{AUC}_{0-24}$, and elimination halflife $\left(t_{1 / 2}\right)$ of single-dose TGZ were comparable between WT and KO rats (Table 2). Repeated TGZ doses had minimal effects on the pharmacokinetics of TGZ, as none of the parameters were significantly different between day 1 and day 7 in either WT or KO rats. In contrast, the $\mathrm{C}_{\max }$ and $\mathrm{AUC}_{0-24}$ of $\mathrm{TS}$ in plasma were significantly decreased in $\mathrm{KO}$ rats, about 7.6- and 




B

TS

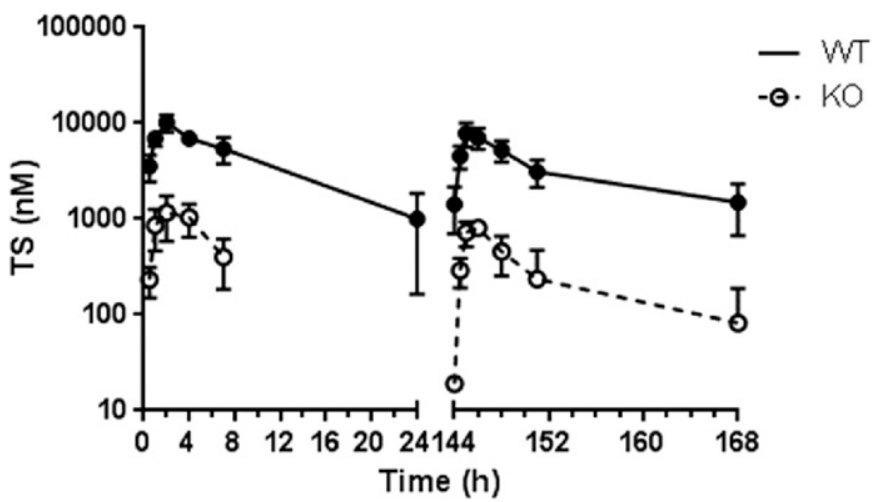

Fig. 1. Plasma concentrations of TGZ (A) and TS (B) on day 1 and day 7 in WT and KO rats following daily oral dose of TGZ (200 mg/kg/day) for 7 days (mean \pm S.D., $n=4-6$ ).

17.9-fold lower than in WT rats on day 1 (Fig. 1B). Similar decreases of TS in the plasma were also observed on day 7 in $\mathrm{KO}$ rats.

At the end of the experiments ( 24 hours after the last dose), liver samples were collected, and the concentrations of TGZ and TS in the liver were measured. Although the liver concentrations of TGZ in both WT and KO rats were below the lower limit of quantitation (30 nM) (Fig. 2A), a substantial amount of TS was still detectable. Similar to the patterns observed in plasma, the liver concentrations of TS in $\mathrm{KO}$ rats were significantly lower than in WT rats. To determine whether the formation of TS is impacted by Bsep KO in rats, TGZ was incubated with liver S9 fractions from WT and KO rats. As shown in Fig. 2B, the formation rates of TS in liver fractions were not different between WT and $\mathrm{KO}$ following incubation at $5,15,30$, and 60 minutes.

To confirm the genetic modification, Bsep mRNA expression was determined using real-time PCR in rat liver samples. When applying the probes targeting the ZFN modification region, Bsep mRNA expression was only detectable in WT rats, not in KO animals (Fig. 3A; Supplemental Fig. 1A). On the other hand, the modified mRNA with the deletion of eight nucleotides was only detected in KO rats (data not shown). Interestingly, a higher amount of Bsep mRNA expression was observed in KO rats than in WT rats when the PCR was designed for the downstream area of the ZFN target mutation region (Supplemental Fig. 1B). In both WT and KO rats, TGZ has no effect on Bsep expression (Supplemental Fig. 1A). Furthermore, the cDNA of Bsep from both WT and KO rats was sequenced, and it was confirmed that the eight nucleotides were removed in all studied KO rats. During the sequencing of rat full-length Bsep cDNA, a splice variant isoform of Bsep was identified in both WT and KO rats, which was a 49-basepair fragment spliced at $3^{\prime}$ of exon 3 compared to the reference sequence NM_031760 (Supplemental Fig. 2).

The gene expressions of major liver transporters [multidrug resistance-associated protein 2 (Mrp2), Mrp3, Mrp4, breast cancer resistance protein (Bcrp), Mdr1, sodium taurocholate cotransporting polypeptide (Ntcp), Oatp1a1] and enzymes/nuclear receptors [farnesoid X receptor ( Fxr), small heterodimer partner (Shp), bile acid-CoA:amino acid $N$-acyltransferase (Baat), Cyp7A1, Sult2a1] were also characterized (Fig. 3). In the rats administered vehicle, the mRNA levels of Mrp2, Mrp3, Mdr1, Baat, Ntcp, Shp, and Sult2A1 in KO rats were significantly increased, whereas Bcrp was decreased when

TABLE 2

Pharmacokinetic parameters of TGZ and TS in WT and Bsep KO rats on day 1 and day 7 following daily oral dose of TGZ $(200 \mathrm{mg} / \mathrm{kg} / \mathrm{day}, 2 \mathrm{ml} / \mathrm{kg} /$ day $)$

Statistical analysis were conducted with one-way analysis of variance. Values are the mean \pm S.D. $(n=4-6)$.

\begin{tabular}{|c|c|c|c|c|}
\hline & $\mathrm{T}_{\max }$ & $\mathrm{C}_{\max }$ & $\mathrm{AUC}_{0-24}$ & $t_{1 / 2}$ \\
\hline & $h$ & $n M$ & $n M^{*} h$ & $h$ \\
\hline \multicolumn{5}{|l|}{ TGZ } \\
\hline \multicolumn{5}{|l|}{ WT } \\
\hline Day 1 & $1.80 \pm 0.45$ & $2080 \pm 343$ & $15,264 \pm 1686$ & $9.00 \pm 7.96$ \\
\hline Day 7 & $1.00^{\mathrm{a}}$ & $2605 \pm 364$ & $15,926 \pm 3602$ & $6.34 \pm 2.02$ \\
\hline \multicolumn{5}{|l|}{ KO } \\
\hline Day 1 & $1.50 \pm 1.22$ & $2720 \pm 721$ & $12,293 \pm 3871$ & $3.41 \pm 1.61$ \\
\hline Day 7 & $1.13 \pm 0.76$ & $2013 \pm 350$ & $11,508 \pm 6699$ & $4.19 \pm 3.64$ \\
\hline \multicolumn{5}{|l|}{ TS } \\
\hline \multicolumn{5}{|l|}{ WT } \\
\hline Day 1 & $2.00^{a}$ & $10,186 \pm 2088$ & $10,2575 \pm 17,140$ & $7.70 \pm 4.62$ \\
\hline Day 7 & $1.00^{a}$ & $7843 \pm 2214$ & $76,269 \pm 23,501$ & $11.13 \pm 3.16$ \\
\hline \multicolumn{5}{|c|}{, } \\
\hline Day 1 & $3.00 \pm 1.10$ & $1335 \pm 470 * * *$ & $5720 \pm 1396^{* * * *}$ & $2.14 \pm 0.52^{*}$ \\
\hline Day 7 & $1.50 \pm 0.58^{\#}$ & $846 \pm 72^{* * *}$ & $6338 \pm 2907^{* * *}$ & $5.92 \pm 0.32^{\# \#}$ \\
\hline
\end{tabular}

${ }^{a}$ S.D. is not available as values are the same among individuals.

${ }^{*} P<0.05,{ }^{* *} P<0.01$, and $* * * P<0.001$ when compared with WT rats on the same day; ${ }^{\#} P<0.05$, and ${ }^{\# \#} P<0.01$ when compared with day 1 in the same rat type. 

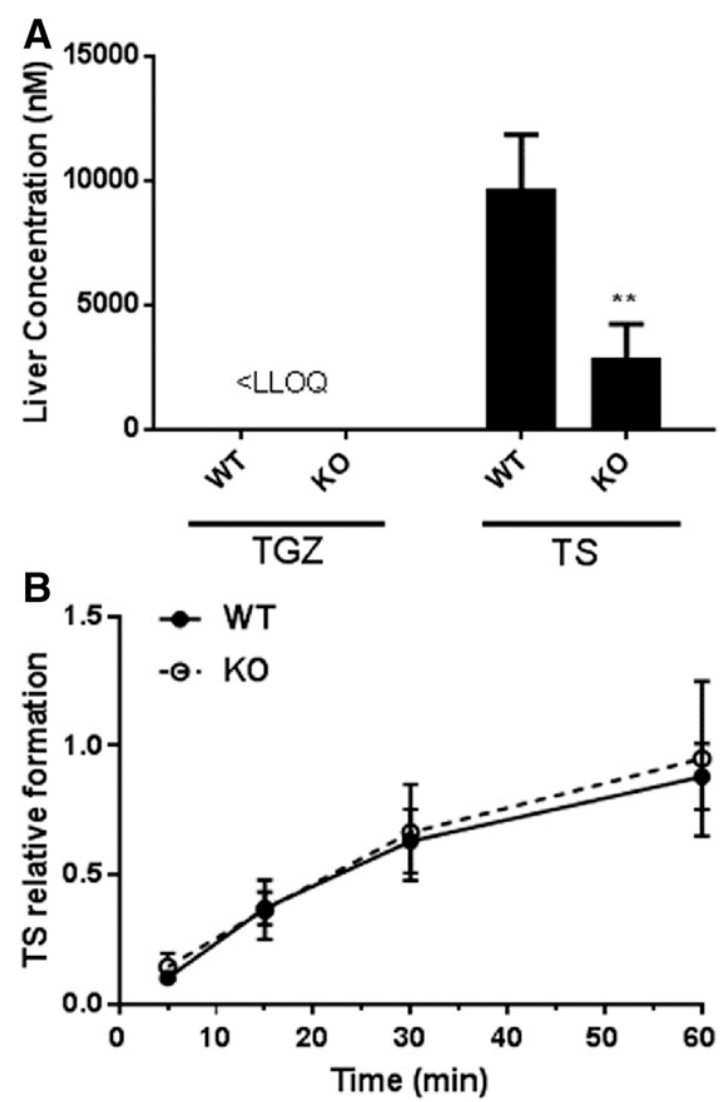

Fig. 2. Liver concentration and the in vitro formation of TS. (A) Liver concentrations of TGZ and TS on day 7 in WT and KO rats following daily oral dose of TGZ (200 mg/kg/day) for 7 days. (B) Relative formation of TS (peak area ratio of TS over internal standard) in liver S9 prepared from $\mathrm{WT}$ and KO rats in the vehicle treatment group. Data are presented as the mean \pm S.D. $(n=5-6) .{ }^{*} P<0.01$ when compared with WT rats. LLOQ, lower limit of quantitation.

compared with WT rats. In both WT and KO rats, repeated doses of TGZ ( $200 \mathrm{mg} / \mathrm{kg} /$ day, 7 days) had no impact on the expression of monitored genes, except that Cyp7A1 was significantly increased.

Bsep KO had no effect on the evaluated serum chemistry parameters, as no changes were observed between WT and KO rats administered the dosing vehicle (Table 3). When dosed with TGZ for 7 days, both WT and KO rats had slightly increased ALT, but the changes were less than 1.5-fold in both cases. All other values, which included liver function indicators (aspartate transaminase, alkaline phosphatase, and total bilirubin), as well as the kidney function markers (blood urea nitrogen and creatinine), were not significantly different between WT and KO rats, between TGZ and vehicle treatment, or between day 1 and day 7 .

The plasma profiles of 13 bile acids were evaluated in WT and KO rats dosed with vehicle or TGZ on day 1 and day 7 (Supplemental Fig. 3). The concentrations of liver bile acids were also quantified at the end of experiments. In animals treated with vehicle control, the majority of the bile acids measured in the plasma displayed similar profiles between day 1 and day 7 in WT rats, except that DCA was slightly reduced, and TCDCA and T $\alpha$ MCA were increased. Similarly, the bile acid profile of $\mathrm{KO}$ rats on day 1 appeared to be the same as that on day 7 (Fig. 4A). The Bsep KO had complicated impacts on bile acid profiles as compared with WT rats. Plasma exposures of GCA, GDCA, GCDCA and TDCA were significantly decreased on both Day-1 and Day-7, while T $\beta$ MCA was significantly increased by Bsep KO. TGZ administration had differential impacts on plasma bile acid levels between WT and KO rats. Following the first TGZ dose in WT rats, the plasma exposures of CA, DCA, $\beta \mathrm{MCA}, \mathrm{T} \beta \mathrm{MCA}$ were significantly higher than that in the rats treated with vehicle (Fig. 4B). Interestingly, repeated TGZ administration attenuated the changes of these bile acids as their concentrations returned to levels comparable to the vehicle group. On the other hand, GCA, GDCA, GCDCA, TCDCA, and T $\alpha$ MCA were significantly increased only in WT rats with repeated TGZ doses. In KO rats, a single dose of TGZ had minimal effects on the bile acid profiles, as compared with vehicle control in $\mathrm{KO}$ rats (Fig. 4C). However, CA, TDCA, TCDCA, $\alpha$ MCA, T $\alpha$ MCA, and $\mathrm{T} \beta \mathrm{MCA}$ in plasma of KO rats were significantly altered by repeated doses of TGZ. In liver, the impact of Bsep KO on bile acid concentrations followed a movement similar to that observed in the plasma (Fig. 4D). Following repeated TGZ treatment, liver concentrations of CA, DCA, CDCA, and $\beta \mathrm{MCA}$ in WT rats tended to increase. In KO rats, only T $\beta$ MCA was significantly increased with repeated TGZ doses.

\section{Discussion}

Bile acids are a class of steroid acids, which are primarily synthesized in the liver and excreted into bile to aid the absorption of fats, lipids, and similar nutrients in the small intestine (Russell, 2003). The biliary secreted bile acids are further metabolized by gut microbiota, and the majority of secreted bile acids are reabsorbed in the ileum and recycled back to the body. Bile acid synthesis is a major route for body cholesterol elimination. Meanwhile, bile acids are the driving force for bile flow, which is important for the elimination of metabolic wastes, such as bilirubin (Kullak-Ublick et al., 2000). Due to the physicochemical characteristics, bile acids possess low membrane permeability in general and require the assistance of transporters to cross the cell membrane. Therefore, interruption of bile acid transport can have severe clinical consequences, such as hyperlipidemia, cholestasis, cholecystitis, and diarrhea, depending on the bile acid transport site of the interruption (Li and Chiang, 2012; Chiang, 2013). As BSEP is the key regulator secreting bile acids in the liver, interference with BSEP activity is expected to influence the body's bile acid profiles.

In the present studies, the Bsep KO rats were developed using zinc finger nuclease technology to delete eight nucleotides from the Bsep gene. This deletion is expected to generate a stop codon on exon 5 and to result in a premature termination of transcription and, subsequently, a functionally deficient Bsep protein. As confirmed by real-time PCR, no Bsep mRNA was detected in the liver of KO rats when applying PCR probes specifically targeting the deleted nucleotides, indicating the successful removal of the intended region. The full cDNA sequencing data further confirmed our gene expression results showing that eight nucleotides were deleted in the Bsep gene in $\mathrm{KO}$ animals. Of interest, while sequencing the Bsep gene in both WT and KO rats, we identified a splice variant form missing 49 nucleotides at exon 3 (Supplemental Fig. 2). Although mutation was not determined on the ZFN-targeting region, it is possible that the 


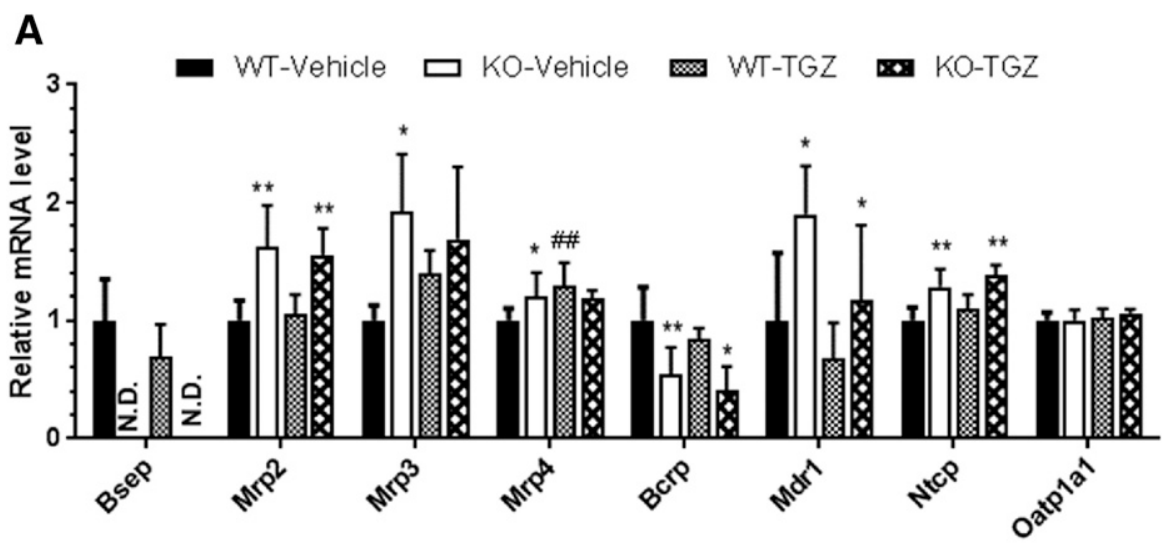

B

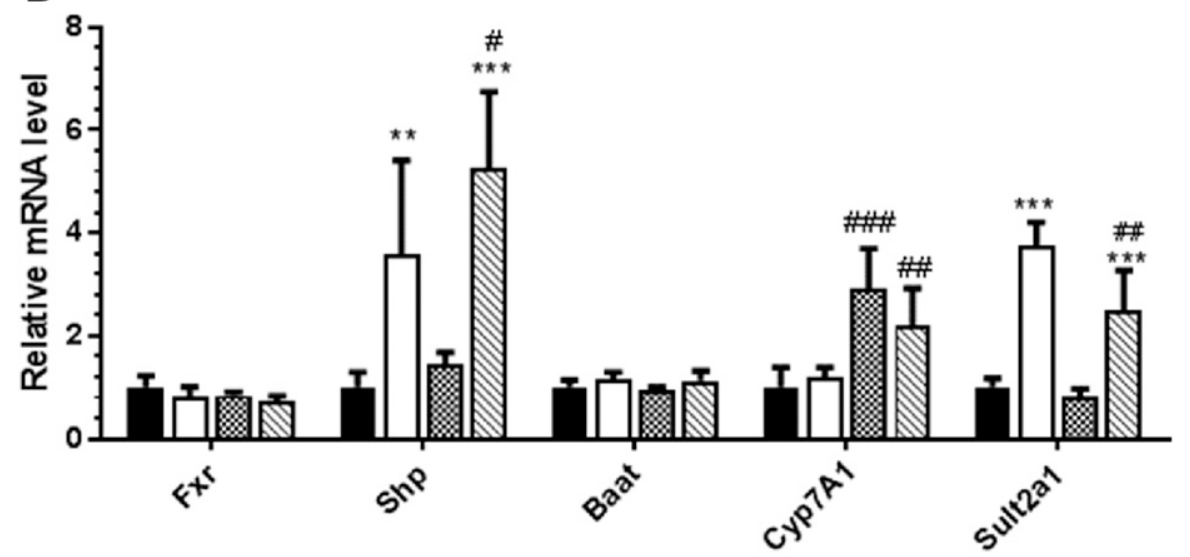

Fig. 3. Relative gene expression of liver transporters (A) and enzymes/nuclear receptors (B) in liver samples from WT and $\mathrm{KO}$ rats administered a daily oral dose of vehicle or TGZ $(200 \mathrm{mg} / \mathrm{kg} /$ day $)$ for 7 days. Data are presented as the mean \pm S.D. $(n=5-6) * P<0.05, * * P<0.01$, and $* * * P<0.001$ when compared with WT rats in the same treatment group; ${ }^{\#} P<0.05,{ }^{\# \#} P<0.01$, and ${ }^{\# \# \#} P<0.001$ when compared with vehicle treatment in the same rat type. N.D., not detected. previously detected trace amount of Bsep protein using the liquid chromatography-tandem mass spectrometry quantitation method could be from an unidentified variant of reported Bsep gene (National Center for Biotechnology Information reference sequence: NM_031760), which was not disrupted in this KO model (Cheng et al., 2016b).

Bsep KO had a substantial impact on the expression of liver transporters in rats. A significantly higher amount of Bsep mRNA expression was observed in $\mathrm{KO}$ rats than in WT rats using the probes targeting outside of the ZFN modification region, representing a feedback response of reduced Bsep function in KO rats. The expression of Mrp2, Mrp3, Mrp4, P-glycoprotein, Bcrp, and Ntcp genes was altered in the liver of $\mathrm{KO}$ rats. As a result, the pre-existing gene regulations of liver transporters impacted the disposition of orally administered TGZ in Bsep KO rats. TGZ is known to be metabolized to TS, TGZ glucuronide, and TGZ quinone in rats and humans, of which TS is the predominant metabolite (Kawai et al., 1997;

TABLE 3

Serum chemistry profiles in WT and Bsep KO rats before administering (predose) or after administering (postdose) seven consecutive daily doses of TGZ ( $200 \mathrm{mg} / \mathrm{kg} / \mathrm{day}, 2 \mathrm{ml} / \mathrm{kg} / \mathrm{day})$ or vehicle control $(2 \mathrm{ml} / \mathrm{kg} / \mathrm{day})$

Values are the mean \pm S.D. $(n=4-5)$.

\begin{tabular}{|c|c|c|c|c|c|c|c|c|}
\hline & AST & ALT & ALP & TBIL & BUN & Creatinine & Cholesterol & Albumin \\
\hline & $U / l$ & $U / l$ & $U / l$ & $m g / d l$ & $m g / d l$ & $m g / d l$ & $m g / d l$ & $m g / d l$ \\
\hline \multicolumn{9}{|l|}{ WT } \\
\hline \multicolumn{9}{|l|}{ Vehicle } \\
\hline Predose & $61.8 \pm 2.95$ & $54.8 \pm 2.17$ & $294 \pm 63.6$ & $<0.10^{\mathrm{a}}$ & $12.4 \pm 0.55$ & $0.23 \pm 0.01$ & $89.4 \pm 7.44$ & $3.60 \pm 0.07$ \\
\hline Postdose & $77.4 \pm 7.13$ & $64.6 \pm 5.98$ & $312 \pm 60.8$ & $<0.10$ & $16.0 \pm 1.41$ & $0.27 \pm 0.00$ & $96.6 \pm 2.07$ & $3.72 \pm 0.13$ \\
\hline \multicolumn{9}{|c|}{ (1) } \\
\hline Predose & $66.8 \pm 2.28$ & $49.4 \pm 7.09$ & $260 \pm 25.8$ & $<0.10$ & $16.6 \pm 1.82$ & $0.28 \pm 0.01$ & $111 \pm 6.52$ & $3.64 \pm 0.18$ \\
\hline Postdose & $70.5 \pm 4.43$ & $70.5 \pm 4.43$ & $252 \pm 14.4$ & $<0.10$ & $16.0 \pm 1.41$ & $0.28 \pm 0.01$ & $76.8 \pm 2.87$ & $3.25 \pm 0.13$ \\
\hline \multicolumn{9}{|l|}{$\mathrm{KO}$} \\
\hline \multicolumn{9}{|l|}{ Vehicle } \\
\hline Predose & $62.8 \pm 5.22$ & $45.4 \pm 5.37$ & $299 \pm 43.6$ & $<0.10$ & $13.6 \pm 1.67$ & $0.25 \pm 0.00$ & $92.8 \pm 8.87$ & $3.68 \pm 0.11$ \\
\hline Postdose & $74.8 \pm 9.50$ & $49.4 \pm 5.41$ & $273 \pm 52.9$ & $<0.10$ & $15.0 \pm 0.71$ & $0.27 \pm 0.01$ & $87.6 \pm 11.2$ & $3.40 \pm 0.14$ \\
\hline \multicolumn{9}{|l|}{ TGZ } \\
\hline Predose & $64.0 \pm 6.04$ & $49.2 \pm 8.04$ & $245 \pm 47.4$ & $<0.10$ & $14.4 \pm 2.88$ & $0.28 \pm 0.01$ & $97.4 \pm 4.56$ & $3.42 \pm 0.08$ \\
\hline Postdose & $70.0 \pm 8.46$ & $65.4 \pm 11.19$ & $248 \pm 55.3$ & $<0.10$ & $15.0 \pm 2.24$ & $0.29 \pm 0.01$ & $88.0 \pm 6.24$ & $3.42 \pm 0.08$ \\
\hline
\end{tabular}

ALP, alkaline phosphatase; AST, aspartate transaminase; BUN, blood urea nitrogen; TBIL, total bilirubin.

${ }^{a}$ Values below the lower limit of quantitation $(0.10 \mathrm{mg} / \mathrm{dl})$. 
A
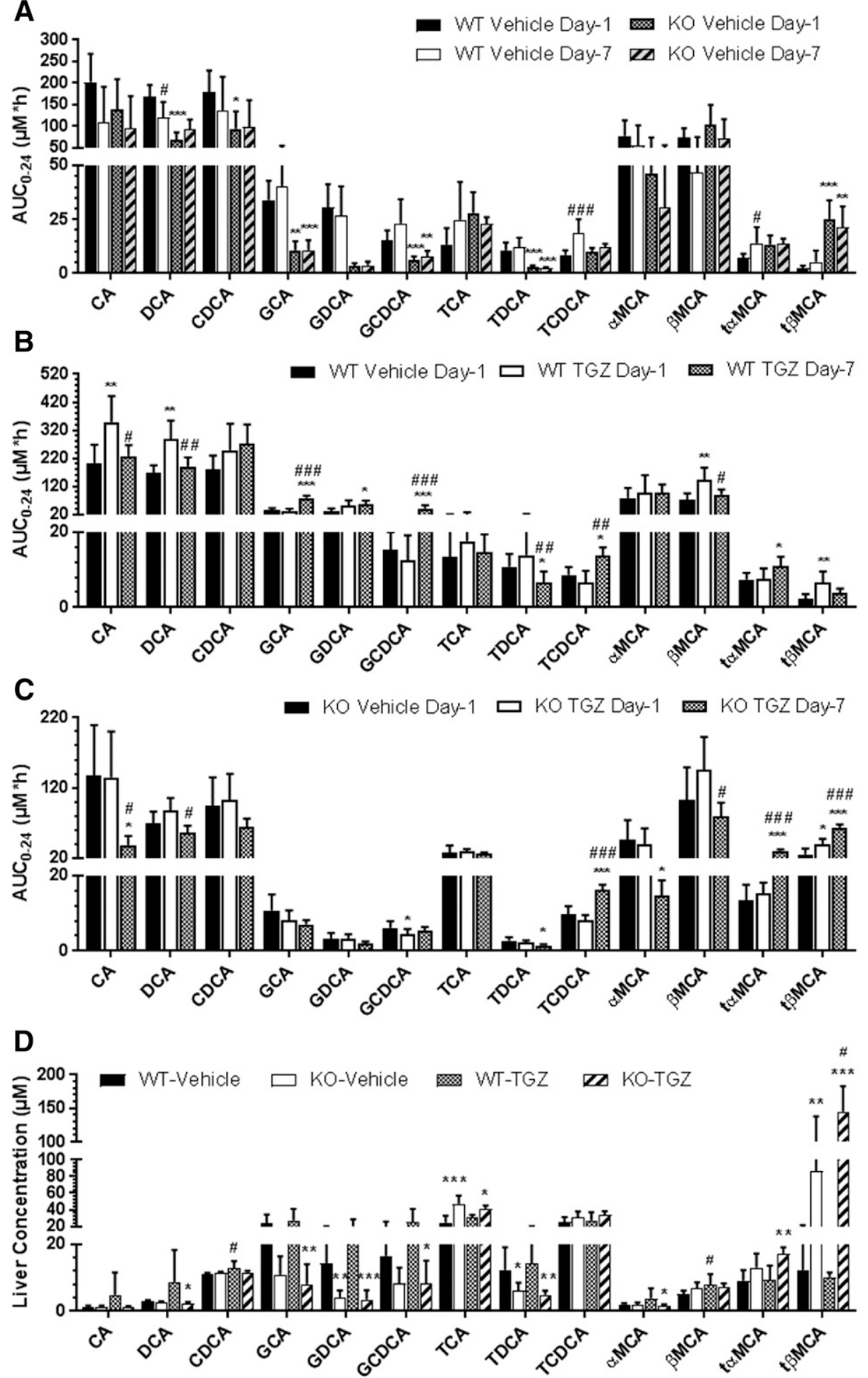

Fig. 4. Individual bile acid profiles in plasma and liver. (A) Plasma exposures $\left(\mathrm{AUC}_{0-24}\right)$ in WT and KO rats dosed with vehicle solution. (B) Plasma exposures $\left(\mathrm{AUC}_{0-24}\right)$ in WT rats administered a single or seven daily oral doses of TGZ ( $200 \mathrm{mg} / \mathrm{kg} /$ day). (C) Plasma exposures $\left(\mathrm{AUC}_{0-24}\right)$ in $\mathrm{KO}$ rats administered a single or seven daily oral doses of TGZ ( $200 \mathrm{mg} / \mathrm{kg} /$ day). (D) Liver concentrations on day 7. Data are presented as the mean \pm S.D. $(n=4-6)$. $* P<$ $0.05, * * P<0.01$, and $* * * P<0.001$ when compared with WT rats in the same treatment group; ${ }^{\#} P<0.05,{ }^{\# \#} P<0.01$, and ${ }^{\# \# \#} P<0.001$ when compared with vehicle treatment in the same rat type.
Loi et al., 1999). In this study, we observed no difference in the pharmacokinetic profiles of TGZ between WT and KO rats and between single and repeated doses, suggesting that transporter regulation detected in $\mathrm{KO}$ rats had no impact on TGZ disposition. However, plasma exposure of TS in KO rats was 7.6- and 9.3-fold lower than in WT rats on day 1 and day 7, respectively. Given the fact that plasma clearance rates of TGZ were comparable between WT and KO rats, the lower TS exposure in $\mathrm{KO}$ rats is not likely due to reduced metabolism, but rather from increased hepatic secretion by upregulating hepatobiliary efflux transporters. This hypothesis was supported by the in vitro data that formations of TS were similar in liver S9 fractions prepared from WT and KO rats. Furthermore, the decrease in TS exposure was consistent with the increased Mrp2 expression in the liver of KO rats, as Mrp2 is the transporter for TS biliary excretion (Kostrubsky et al., 
2001). Therefore, caution should be taken when using KO animals in drug pharmacokinetic and toxicity evaluations since regulation of other transporters and factors could occur to compensate for the absence of the interested transporter, which could complicate the disposition of tested drugs and then lead to misguided conclusions.

The impairment of Bsep function is expected to produce an accumulation of bile acids in hepatocytes, which will activate the nuclear receptor Fxr and then the downstream regulator Shp, leading to the upregulation of efflux transporter genes and downregulation of bile acid uptake and synthesis (Tu et al., 2000; Poupon, 2012). In this study, impairment of Bsep activity by Bsep KO and repeated TGZ treatment had sophisticated and differential regulations of liver transporters, enzymes, and gene regulators that are associated with bile acid homeostasis, as illustrated in Fig. 5. It should be noted that the evaluation of gene expression was conducted at the end of the experiments, 24 hours after the last dose; therefore, the monitored gene expression reflects the overall changes including the compensatory effects following repeated doses. Nevertheless, the gene changes generally agree with previous reports, except for Cyp7A1 (Poupon, 2012).

Cyp7A1 is the rate-limiting enzyme in bile acid synthesis from cholesterol and is suppressed by Shp, which is induced by Fxr activation (Goodwin et al., 2000; Rodrigues et al., 2014). Surprisingly, the expression of Cyp7A1 was significantly upregulated in WT and KO rats with repeated TGZ administration, although the level seemed to be slightly suppressed in KO rats compared with WT rats, probably as a result of higher Shp in KO animals. The mechanism for this Cyp7A1 upregulation remains unknown. One possible explanation could be that repeated TGZ administration complicated the regulation of Cyp7A1 through the engagement of other pathways, i.e., the activation of the peroxisome proliferator-activated receptors (PPARs). In fact, TGZ is a potent agonist of PPAR $\gamma$ developed for the treatment of diabetic and inflammatory disease (Escher and Wahli, 2000; Coyle and Kinsella, 2006). It was reported that activation of PPAR $\gamma$ could induce CYP7A1 expression to enhance cholesterol metabolism (Duan et al., 2012). Further studies are necessary to understand the complicated mechanism of TGZ effects on Cyp7A1 expression. Since the bile acid pool in humans is more hydrophobic and toxic, the induced Cyp7A1 expression by TGZ may increase bile acid production and raise the liver load of bile acid accumulation and stress when a compensatory mechanism is not sufficient.

The changes of transporters, enzymes, and regulators following Bsep impairment are correlated with the observed bile acid alterations. Following a single dose of TGZ, plasma exposures of CA, DCA, $\beta \mathrm{MCA}$, and T $\beta$ MCA were significantly increased in WT rats. In contrast, the single dose of TGZ had no effect in Bsep KO rats. The differential impacts of single TGZ administration between KO and WT rats indicate that the increase of bile acids in WT rats could be due to Bsep inhibition by TGZ and/or TS. In fact, the average liver concentration of TS was $9.7 \mu \mathrm{M}$ in WT rats at 24 hours after the last dose, with a liver to plasma ratio of 7.7. Assuming that this ratio is consistent and the intracellular unbound fraction of TS is 0.0032 , the predicted maximum free concentration of TS in the liver is $0.25 \mu \mathrm{M}$, slightly greater than the reported TS $\mathrm{IC}_{50}$ value $(0.2 \mu \mathrm{M})$ against rat Bsep (Funk et al., 2001a; Guo et al., 2017). Actually, the maximum liver TS concentration
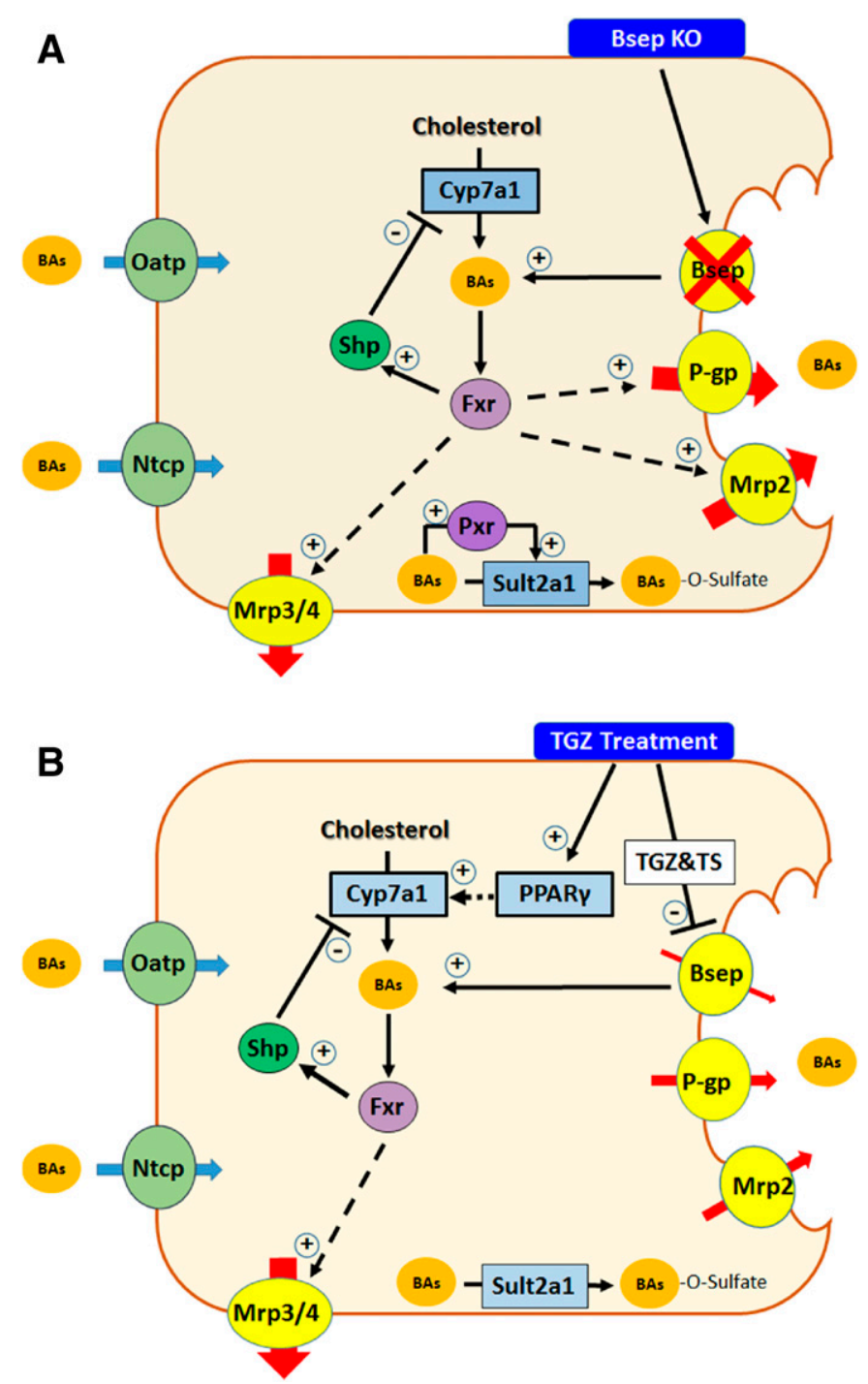

Fig. 5. Proposed regulations of bile acid synthesis and transport in rat liver followed by Bsep KO (A) or TGZ treatment (B). BAs, bile acids; P-gp, P-glycoprotein.

could be higher than this prediction, because the high concentration of TS at $T_{\max }$ may exceed the excretion capacity of Mrp2. Interestingly, the altered plasma concentration of bile acids by a single dose of TGZ was attenuated in WT rats following repeated TGZ doses. The increased plasma exposures of CA, DCA, $\beta$ MCA, and T $\beta$ MCA observed with a single dose of TGZ returned to levels comparable to the control group, indicating the acquired compensatory regulation of bile acid homeostasis followed by the long-term inhibition of Bsep by TGZ/TS. Baat, which catalyzes the formation of glycine- or taurine-conjugated bile acids, remained unchanged in all treatments. Therefore, the observed changes in taurineand glycine-conjugated bile acids in the plasma and liver of KO rats were likely due to altered transporter activity. Meanwhile, TGZ appeared to have no impact on transporter expression, and taurine- and glycine-conjugated bile acids were minimally altered in TGZ-treated rats.

Interference of BSEP function is thought to be one of the mechanisms for TGZ-induced liver injury in humans (Smith, 2003). A mechanism-based systemic pharmacology model demonstrated that serum ALT levels were found to be 
sensitive to the inhibition of BSEP with the dose of TGZ in humans (Yang et al., 2014). Here, we found that the hydrophilic and less-toxic bile acids were changed dramatically by Bsep KO and repeated TGZ treatments in rats, whereas the hydrophobic and cytotoxic bile acids, DCA and CDCA, were minimally affected (Schölmerich et al., 1984; Perez and Briz, 2009). This agrees with the serum chemistry data that no signal of liver toxicity was observed. Regulation of transporters and bile acid compositions seem to be the protective mechanisms for rats against Bsep impairment, and may be a reason why the rat failed to serve as a good preclinical model to predict DILI in humans, especially with respect to BSEP inhibition. However, the observed individual bile acid profiles in plasma seem to correlate with Bsep impairment in rats, and therefore, could be biomarkers to evaluate Bsep function in early drug discovery. It has been reported that the plasma levels of selected bile acids, such as GCA and GCDCA, are strongly associated with OATP1B activity in cynomolgus monkeys and humans (Xiang et al., 2009; Chu et al., 2015; Yang et al., 2016; Yee et al., 2016). Further investigations are required to identify the specific bile acids as biomarkers for BSEP function and to confirm whether the results in rats can be translated to humans.

In summary, the expression of liver transporters was significantly impacted by Bsep $\mathrm{KO}$ in rats, leading to the altered disposition profiles of TGZ sulfate in plasma and liver. Repeated TGZ treatment had a minimal impact on liver transporters, but significantly upregulated Cyp7A1. These compensatory regulations were correlated with the changes of individual bile acids in plasma. Additionally, this is the first report indicating that TGZ can increase Cyp7A1 expression, probably through activation of the PPAR $\gamma$ nuclear receptor, which could be a potential explanation for TGZ-induced liver injury, in addition to the mechanism of BSEP inhibition in humans.

\section{Authorship Contributions}

Participated in research design: Cheng, Humphreys, Gan, Lai.

Conducted experiments: Cheng, S. Chen, Freeden, W. Chen, Zhang, Abraham.

Performed data analysis: Cheng, Gan, Lai.

Wrote or contributed to the writing of the manuscript: Cheng, Nelson, Humphreys, Gan, Lai.

\section{References}

Cheng Y, El-Kattan A, Zhang Y, Ray AS, and Lai Y (2016a) Involvement of Drug Transporters in Organ Toxicity: The Fundamental Basis of Drug Discovery and Development. Chem Res Toxicol 29:545-563.

Cheng Y, Freeden C, Zhang Y, Abraham P, Shen H, Wescott D, Humphreys WG, Gan J, and Lai Y (2016b) Biliary excretion of pravastatin and taurocholate in rats with bile salt export pump (Bsep) impairment. Biopharm Drug Dispos 37:276-286.

Chiang JY (2013) Bile acid metabolism and signaling. Compr Physiol 3:1191-1212.

Chu X, Shih SJ, Shaw R, Hentze H, Chan GH, Owens K, Wang S, Cai X, Newton D, Castro-Perez J, et al. (2015) Evaluation of cynomolgus monkeys for the identification of endogenous biomarkers for hepatic transporter inhibition and as a translatable model to predict pharmacokinetic interactions with statins in humans. Drug Metab Dispos 43:851-863.

Coyle AT and Kinsella BT (2006) Synthetic peroxisome proliferator-activated receptor gamma agonists rosiglitazone and troglitazone suppress transcription by promoter 3 of the human thromboxane A2 receptor gene in human erythroleukemia cells. Biochem Pharmacol 71:1308-1323.

Duan Y, Chen Y, Hu W, Li X, Yang X, Zhou X, Yin Z, Kong D, Yao Z, Hajjar DP, et al. (2012) Peroxisome Proliferator-activated receptor $\gamma$ activation by ligands and dephosphorylation induces proprotein convertase subtilisin kexin type 9 and low density lipoprotein receptor expression. J Biol Chem 287:23667-23677.

Escher P and Wahli W (2000) Peroxisome proliferator-activated receptors: insight into multiple cellular functions. Mutat Res 448:121-138.

Funk C, Pantze M, Jehle L, Ponelle C, Scheuermann G, Lazendic M, and Gasser R (2001a) Troglitazone-induced intrahepatic cholestasis by an interference with the hepatobiliary export of bile acids in male and female rats. Correlation with the gender difference in troglitazone sulfate formation and the inhibition of the canalicular bile salt export pump (Bsep) by troglitazone and troglitazone sulfate. Toxicology 167:83-98.

Funk C, Ponelle C, Scheuermann G, and Pantze M (2001c) Cholestatic potential of troglitazone as a possible factor contributing to troglitazone-induced hepatotoxicity: in vivo and in vitro interaction at the canalicular bile salt export pump (Bsep) in the rat. Mol Pharmacol 59:627-635.

Gerloff T, Stieger B, Hagenbuch B, Madon J, Landmann L, Roth J, Hofmann AF, and Meier PJ (1998) The sister of P-glycoprotein represents the canalicular bile salt export pump of mammalian liver. J Biol Chem 273:10046-10050.

Goodwin B, Jones SA, Price RR, Watson MA, McKee DD, Moore LB, Galardi C, Wilson JG, Lewis MC, Roth ME, et al. (2000) A regulatory cascade of the nuclear receptors FXR, SHP-1, and LRH-1 represses bile acid biosynthesis. Mol Cell 6: $517-526$

Guo C, Yang K, Liao M, Xia CQ, Brouwer KR, and Brouwer KLR (2017) Prediction of hepatic efflux transporter-mediated drug interactions: when is it optimal to measure intracellular unbound fraction of inhibitors? J Pharm Sci DOI: 10.1016/j. xphs.2017.04.054 [published ahead of print].

Ho RH, Leake BF, Kilkenny DM, Meyer Zu Schwabedissen HE, Glaeser H, Kroetz DL, and Kim RB (2010) Polymorphic variants in the human bile salt export pump (BSEP; ABCB11): functional characterization and interindividual variability. Pharmacogenet Genomics 20:45-57.

Hussaini SH and Farrington EA (2007) Idiosyncratic drug-induced liver injury: an overview. Expert Opin Drug Saf 6:673-684.

Izumi T, Enomoto S, Hosiyama K, Sasahara K, Shibukawa A, Nakagawa T, and Sugiyama Y (1996) Prediction of the human pharmacokinetics of troglitazone, a new and extensively metabolized antidiabetic agent, after oral administration, with an animal scale-up approach. J Pharmacol Exp Ther 277:1630-1641.

Jansen PL, Strautnieks SS, Jacquemin E, Hadchouel M, Sokal EM, Hooiveld GJ, Koning JH, De Jager-Krikken A, Kuipers F, Stellaard F, et al. (1999) Hepatocanalicular bile salt export pump deficiency in patients with progressive familial intrahepatic cholestasis. Gastroenterology 117:1370-1379.

Kawai K, Kawasaki-Tokui Y, Odaka T, Tsuruta F, Kazui M, Iwabuchi H, Nakamura T, Kinoshita T, Ikeda T, Yoshioka T, et al. (1997) Disposition and metabolism of the new oral antidiabetic drug troglitazone in rats, mice and dogs. Arzneimittelforschung 47:356-368.

Kostrubsky VE, Vore M, Kindt E, Burliegh J, Rogers K, Peter G, Altrogge D, and Sinz MW (2001) The effect of troglitazone biliary excretion on metabolite distribution and cholestasis in transporter-deficient rats. Drug Metab Dispos 29:1561-1566.

Kullak-Ublick GA, Stieger B, Hagenbuch B, and Meier PJ (2000) Hepatic transport of bile salts. Semin Liver Dis 20:273-292.

Lammert C, Einarsson S, Saha C, Niklasson A, Bjornsson E, and Chalasani N (2008) Relationship between daily dose of oral medications and idiosyncratic druginduced liver injury: search for signals. Hepatology 47:2003-2009.

Lang C, Meier Y, Stieger B, Beuers U, Lang T, Kerb R, Kullak-Ublick GA, Meier PJ, and Pauli-Magnus C (2007) Mutations and polymorphisms in the bile salt export pump and the multidrug resistance protein 3 associated with drug-induced liver injury. Pharmacogenet Genomics 17:47-60.

Leise MD, Poterucha JJ, and Talwalkar JA (2014) Drug-induced liver injury. Mayo Clin Proc 89:95-106.

Li T and Chiang JY (2012) Bile Acid signaling in liver metabolism and diseases. J Lipids 2012:754067.

Loi CM, Alvey CW, Vassos AB, Randinitis EJ, Sedman AJ, and Koup JR (1999) Steady-state pharmacokinetics and dose proportionality of troglitazone and its metabolites. J Clin Pharmacol 39:920-926.

Meier PJ, Sztul ES, Reuben A, and Boyer JL (1984) Structural and functional polarity of canalicular and basolateral plasma membrane vesicles isolated in high yield from rat liver. J Cell Biol 98:991-1000.

Morgan RE, Trauner M, van Staden CJ, Lee PH, Ramachandran B, Eschenberg M, Afshari CA, Qualls, Jr CW, Lightfoot-Dunn R, and Hamadeh HK (2010) Interference with bile salt export pump function is a susceptibility factor for human liver injury in drug development. Toxicol Sci 118:485-500.

Morgan RE, van Staden CJ, Chen Y, Kalyanaraman N, Kalanzi J, Dunn, 2nd RT, Afshari CA, and Hamadeh HK (2013) A multifactorial approach to hepatobiliary transporter assessment enables improved therapeutic compound development. Toxicol Sci 136:216-241.

Noe J, Hagenbuch B, Meier PJ, and St-Pierre MV (2001) Characterization of the mouse bile salt export pump overexpressed in the baculovirus system. Hepatology 33:1223-1231.

Pedersen JM, Matsson P, Bergström CA, Hoogstraate J, Norén A, LeCluyse EL, and Artursson P (2013) Early identification of clinically relevant drug interactions with the human bile salt export pump (BSEP/ABCB11). Toxicol Sci 136:328-343.

Perez MJ and Briz O (2009) Bile-acid-induced cell injury and protection. World $J$ Gastroenterol 15:1677-1689.

Perwaiz S, Forrest D, Mignault D, Tuchweber B, Phillip MJ, Wang R, Ling V, and Yousef IM (2003) Appearance of atypical 3 alpha, 6 beta,7 beta,12 alphatetrahydroxy-5 beta-cholan-24-oic acid in spgp knockout mice. J Lipid Res 44: 494-502.

Poupon R (2012) Ursodeoxycholic acid and bile-acid mimetics as therapeutic agents for cholestatic liver diseases: an overview of their mechanisms of action. Clin Res Hepatol Gastroenterol 36 (Suppl 1):S3-S12.

Rodrigues AD, Lai Y, Cvijic ME, Elkin LL, Zvyaga T, and Soars MG (2014) Druginduced perturbations of the bile acid pool, cholestasis, and hepatotoxicity: mechanistic considerations beyond the direct inhibition of the bile salt export pump. Drug Metab Dispos 42:566-574.

Russell DW (2003) The enzymes, regulation, and genetics of bile acid synthesis. Annu Rev Biochem 72:137-174.

Schölmerich J, Becher MS, Schmidt K, Schubert R, Kremer B, Feldhaus S, and Gerok W (1984) Influence of hydroxylation and conjugation of bile salts on their membrane-damaging properties-studies on isolated hepatocytes and lipid membrane vesicles. Hepatology 4:661-666. 
Smith MT (2003) Mechanisms of troglitazone hepatotoxicity. Chem Res Toxicol 16: 679-687.

Stieger B, O'Neill B, and Meier PJ (1992) ATP-dependent bile-salt transport in canalicular rat liver plasma-membrane vesicles. Biochem $J$ 284:67-74.

Tu H, Okamoto AY, and Shan B (2000) FXR, a bile acid receptor and biological sensor. Trends Cardiovasc Med 10:30-35.

Wang R, Chen HL, Liu L, Sheps JA, Phillips MJ, and Ling V (2009) Compensatory role of P-glycoproteins in knockout mice lacking the bile salt export pump. Hepatology 50:948-956.

Wang R, Salem M, Yousef IM, Tuchweber B, Lam P, Childs SJ, Helgason CD Ackerley C, Phillips MJ, and Ling V (2001) Targeted inactivation of sister of $\mathrm{P}$-glycoprotein gene (spgp) in mice results in nonprogressive but persistent intrahepatic cholestasis. Proc Natl Acad Sci USA 98:2011-2016.

Xiang X, Han Y, Neuvonen M, Pasanen MK, Kalliokoski A, Backman JT, Laitila J, Neuvonen PJ, and Niemi M (2009) Effect of SLCO1B1 polymorphism on the plasma concentrations of bile acids and bile acid synthesis marker in humans. Pharmacogenet Genomics 19:447-457.
Yang K, Guo C, Woodhead JL, St Claire, 3rd RL, Watkins PB, Siler SQ, Howell BA, and Brouwer KL (2016) Sandwich-Cultured Hepatocytes as a Tool to Study Drug Disposition and Drug-Induced Liver Injury. J Pharm Sci 105:443-459.

Yang K, Woodhead JL, Watkins PB, Howell BA, and Brouwer KL (2014) Systems pharmacology modeling predicts delayed presentation and species differences in bile acid-mediated troglitazone hepatotoxicity. Clin Pharmacol Ther 96: $589-598$.

Yee SW, Giacomini MM, Hsueh CH, Weitz D, Liang X, Goswami S, Kinchen JM, Coelho A, Zur AA, Mertsch K, et al. (2016) Metabolomic and Genome-wide Association Studies Reveal Potential Endogenous Biomarkers for OATP1B1. Clin Pharmacol Ther 100:524-536.

Address correspondence to: Dr. Yaofeng Cheng, Pharmaceutical Candidate Optimization, Bristol-Myers Squibb, P.O. Box 4000, Princeton, NJ 08543. E-mail: yaofeng.cheng@BMS.com 